\title{
Reduced-Port Laparoscopic Surgery for a Tumor-Specific Mesorectal Excision in Patients With Colorectal Cancer: Initial Experience With 20 Consecutive Cases
}

\author{
Sung Uk Bae, Se Jin Baek ${ }^{1}$, Byung Soh Min ${ }^{1}$, Seung Hyuk Baik ${ }^{1}$, Nam Kyu Kim , Hyuk Hur ${ }^{1}$ \\ Division of Colorectal Surgery, Department of Surgery, Keimyung University Dongsan Medical Center, Keimyung University School of \\ Medicine, Daegu; ${ }^{1}$ Division of Colorectal Surgery, Department of Surgery, Colorectal Cancer Clinic, Severance Hospital, Yonsei University \\ College of Medicine, Seoul, Korea
}

Purpose: Single-port plus one-port, reduced-port laparoscopic surgery (RPLS) may decrease collisions between laparoscopic instruments and the camera in a narrow, bony, pelvic cavity while maintaining the cosmetic advantages of single-incision laparoscopic surgery. The aim of this study is to describe our initial experience with and to assess the feasibility and safety of RPLS for tumor-specific mesorectal excisions (TSMEs) in patients with colorectal cancer.

Methods: Between May 2010 and August 2012, RPLS for TSME was performed in 20 patients with colorectal cancer. A single port with four channels through an umbilical incision and an additional port in the right lower quadrant were used for RPLS.

Results: The median operation time was 231 minutes (range, 160-347 minutes), and the estimated blood loss was $100 \mathrm{~mL}$ (range, 50-500 mL). We transected the rectum with one laparoscopic stapler in 17 cases (85\%). The median time to soft diet was 4 days (range, 3-6 days), and the length of hospital stay was 7 days (range, 5-45 days). The median total number of lymph nodes harvested was 16 (range, 7-36), and circumferential resection margin involvement was found in 1 case (5\%). Seven patients (35\%) developed postoperative complications, and no mortalities occurred within 30 days. During the median follow-up period of 20 months (range, 12-40 months), liver metastasis occurred in 1 patient 10 months after surgery, and local recurrence was nonexistent.

Conclusion: RPLS for TSME in patients with colorectal cancer is technically feasible and safe without compromising oncologic safety. However, further studies comparing RPLS with a conventional, laparoscopic low-anterior resection are needed to prove the advantages of the RPLS procedure.

Keywords: Rectal neoplasms; Natural orifice endoscopic surgery; Laparoscopy

Received: December 17, 2014 - Accepted: January 7, 2015

Correspondence to: Hyuk Hur, M.D.

Division of Colorectal Surgery, Department of Surgery, Colorectal Cancer Clinic, Severance Hospital, Yonsei University College of Medicine, 50 Yonsei-ro, Seodaemun-gu, Seoul 120-752, Korea

Tel: +82-2-2228-2139, Fax: +82-2-313-8289

E-mail: hhyuk@yuhs.ac

*This study was presented at the Forty-Seventh Annual Convention of the Korean Society of Coloproctology, April 11-13, 2014, Muju, Korea.

(C) 2015 The Korean Society of Coloproctology

This is an open-access article distributed under the terms of the Creative Commons Attribution NonCommercial License (http://creativecommons.org/licenses/by-nc/3.0) which permits unrestricted noncommercial use, distribution, and reproduction in any medium, provided the original work is properly cited.

\section{INTRODUCTION}

Laparoscopic surgery for rectal cancer, although technically demanding, has been accepted since some randomized trials [1-5] reported that, compared to open surgery, it had advantages, including less blood loss, earlier return of bowel motility, and shorter hospital stay, without compromising oncologic outcomes. With minimally-invasive surgery becoming increasingly common, efforts are aimed at minimizing morbidity and improving cosmetic outcomes. These concepts have led to the development of singleincision laparoscopic surgery (SILS) for the treatment of a variety of conditions. However, SILS for treating rectal cancer is a challenging procedure, even in the hands of a skilled, laparoscopic, 
colorectal surgeon because of the difficulty in creating triangulation and in the application of laparoscopic staplers in the narrow pelvic cavity.

Single-port plus one-port, reduced-port laparoscopic surgery (RPLS) may decrease collisions between laparoscopic instruments and the camera while maintaining the cosmetic advantages of SILS and may make it possible to transect the lower rectum with pelvic drain placement. A few retrospective studies have focused on the safety and the efficacy of SILS for treating rectal cancer. The aim of this study is to describe our initial experience with and to assess the feasibility and the safety of RPLS for tumor-specific mesorectal excision (TSME) in patients with colorectal cancer.

\section{METHODS}

Between May 2010 and August 2012, RPLS for TSME was performed in 20 patients with colorectal cancer at Severance Hospital, Yonsei University College of Medicine. All cases were performed by a single surgeon (H.H.) who was experienced in conventional laparoscopic surgery, as well as in SILS, for treating colorectal disease. All procedures were performed using a single port with a four-channel system (OCTO Port, Dalim Surgnet, Seoul, Korea). Informed consent was obtained from all patients. Information regarding patient demographics was obtained from a prospectively collected Yonsei Colorectal Cancer Database and included data regarding age, sex, American Society of Anesthesiologists (ASA) grade, history of previous abdominal surgeries, preoperative chemoradiotherapy, type of port, preoperative carcinoembryonic antigen (CEA), and distance of the tumor from the anal verge. Perioperative details included operative time, blood loss, pain score, number of days to first flatus, number of days to soft diet, and number of days to removal of the urinary catheter, length of hospital stay, perioperative complications, and histopathological findings. The location of the tumor was categorized as rectosigmoid colon (distal border of tumor between a location $15 \mathrm{~cm}$ from the anal verge and the rectosigmoid junction), upper (10-15 cm from the anal verge), middle (5-10 $\mathrm{cm}$ from the anal verge), and lower rectum $(<5 \mathrm{~cm}$ from the anal verge).

All patients underwent preoperative evaluation, including history taking, routine laboratory tests, chest radiography, and colonoscopy with biopsy. The level of preoperative serum CEA was measured, and computed tomography (CT) of the abdomen and the pelvis was performed in all patients. In patients with rectal cancer, magnetic resonance imaging of the rectum and transrectal ultrasonography were performed. The selection criteria for preoperative chemoradiotherapy at our institution include a histologicallyproven carcinoma of the mid or distal rectum and a tumor that is clinically staged as T3/T4 or node positive after preoperative evaluation. Preoperative chemoradiotherapy was given using 5-fluorouracil and leucovorin with concurrent radiation. The total radiation dosage was 5,040 cGy in 25 fractions delivered over 5 weeks. Adjuvant chemotherapy was administered 3-4 weeks after surgery.
The indications for a protective defunctioning ileostomy for rectal cancer included male sex, low anastomosis, preoperative chemoradiation, and a positive air-leak test. A protective diverting stoma was formed in patients with two or more of these risks.

Conversion to open surgery was defined as an interruption of the laparoscopic approach followed by the need for a laparotomy, and conversion to multiport surgery was defined as a requirement for the placements of more than two additional ports at any time to complete the surgical procedure. For the evaluation of postoperative pain, the visual analogue scale (VAS) was used. Disease stages were classified according to the American Joint Committee on Cancer Tumor Node Metastasis Classification, 6th edition. The circumferential resection margin (CRM) was considered positive when the distance from the tumor to the mesorectal fascia was 1 $\mathrm{mm}$ or less. Follow-ups on patients were performed routinely at the first and the third months, then every 3 months for 2 years and every 6 months for 5 years. Serum CEA levels were measured at every visit. Chest and abdominopelvic CT scans, taken every six months, were used for local detection of systemic recurrence. Recurrence was defined as the presence of a radiologically and/or histologically confirmed tumor, and the location of recurrence was defined as the first site of recurrence after a complete resection. The policy on adjuvant chemotherapy for patient followed the guidelines of the National Comprehensive Cancer Network. Data were expressed as median (range), and analyses were performed using IBM SPSS Statistics ver. 20.0 (IBM Co., Armonk, NY, USA).

For bowel preparation, colonic lavage using $4 \mathrm{~L}$ of Colyte was performed on the day before the operation. Patients were given prophylactic antibiotics. All surgical procedures were carried out under general anesthesia with the patients in the lithotomy posi-

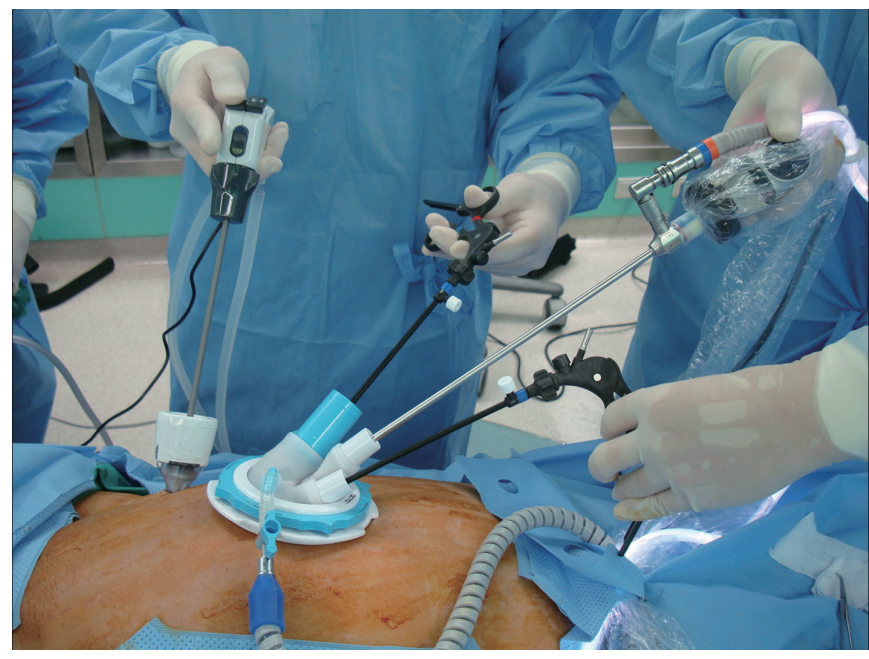

Fig. 1. Trocar and device placement: a 5-mm access channel for the single port and an additional port were used for the operating surgeon, and one of three 5-mm access channels was used for the assistant surgeon. 
tion. Patients were put in the Trendelenburg position at $30^{\circ}$ and were tilted right-side-down at an angle of $10^{\circ}-15^{\circ}$; a systematic rigid sigmoidoscopy was routinely performed in the operating room prior to surgery.

A single $3-\mathrm{cm}$ vertical incision was made through the umbilical skin. The subcutaneous tissue was dissected and deepened into the linea alba. The linea alba was then incised vertically, and the peritoneum was opened. A single port was placed in the abdominal cavity through the umbilical incision. After a pneumoperitoneum with insufflations of $\mathrm{CO}_{2}$ to $12 \mathrm{mmHg}$ had been achieved, a $5-\mathrm{mm}$ diameter telescope with fiberoptic light transmission and $30^{\circ}$ angled view was inserted through a 5-mm channel of the single port. A 12-mm additional port in right lower quadrant was inserted under direct vision (Fig. 1). A 5-mm access channel of the single port, an additional port for the operating surgeon, and one of three 5-mm access channels for the assistant surgeon were used. In this study, no specialized instruments, either bent or flexible, were used.

The peritoneum was incised at the level of the sacral promontory above the bifurcation of the iliac artery. Medial-to-lateral mobilization of the sigmoid and descending colon along the avascular plane was performed, and the left ureter and gonadal vessels were identified (Fig. 2A). After dissection of the lymph nodes around the root of the inferior mesenteric artery, high ligation or low ligation of the inferior mesenteric artery while preserving the left colic branch was performed (Fig. 2B). After the inferior mesenteric vein (IMV) had been raised by the assistant surgeon, the peritoneum under the IMV was incised. A retroperitoneal space between the mesocolon and the retroperitoneal structures, including Gerota's fascia, gonadal vessels, and the ureter, was made. While the descending colon was retracted to the medial side by the assistant surgeon, the left paracolic gutter was dissected, joining the previous surgical plane of the left Toldt fascia. For a tension-free anastomosis, complete splenic flexure mobilization was done in the case of a lack of redundancy of the sigmoid colon. A rectal dissection using TSME principles and autonomic-nerve preservation was performed (Fig. 2C).

One or two endoscopic linear stapling devices were introduced through the additional port, and the rectum was transected (Fig. $2 \mathrm{D}, \mathrm{F})$. Surgical specimens were subsequently removed via a $3-\mathrm{cm}$ transumbilical incision through the single port as a wound protector (Fig. 2F). In some patients, the incision was extended for retrieval of the specimen to a maximum of $4.5 \mathrm{~cm}$, depending on the size of the specimen. The anvil of the circular stapler was inserted into the proximal bowel, and the bowel was placed back into the abdominal cavity. The single port was reintroduced, and the pneumoperitoneum was reestablished. End-to-end intracorporeal anastomosis was carried out using a double-stapling technique with a circular stapler (CDH29, Ethicon Endo-Surgery Inc., Cincinnati, OH, USA). All anastomoses were air-leak tested at the
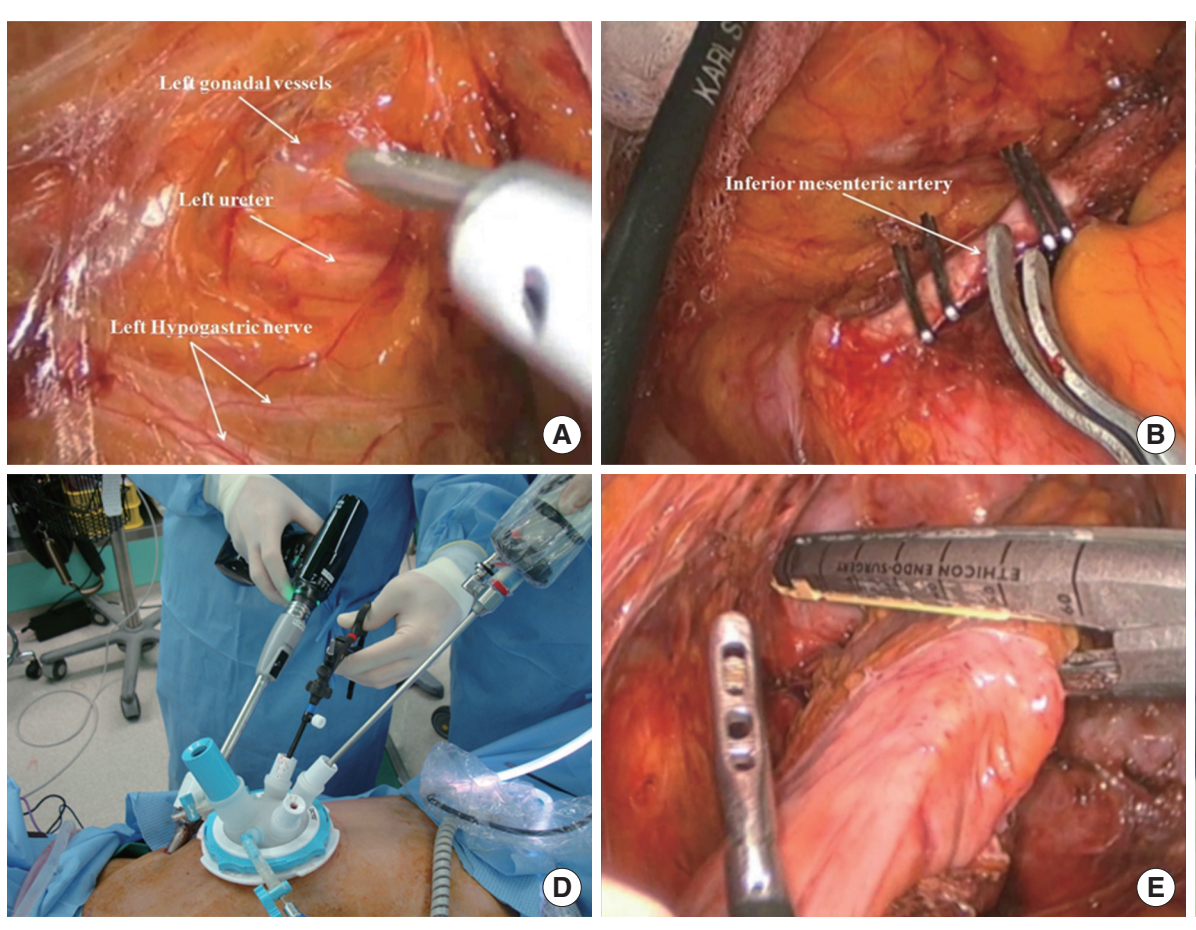
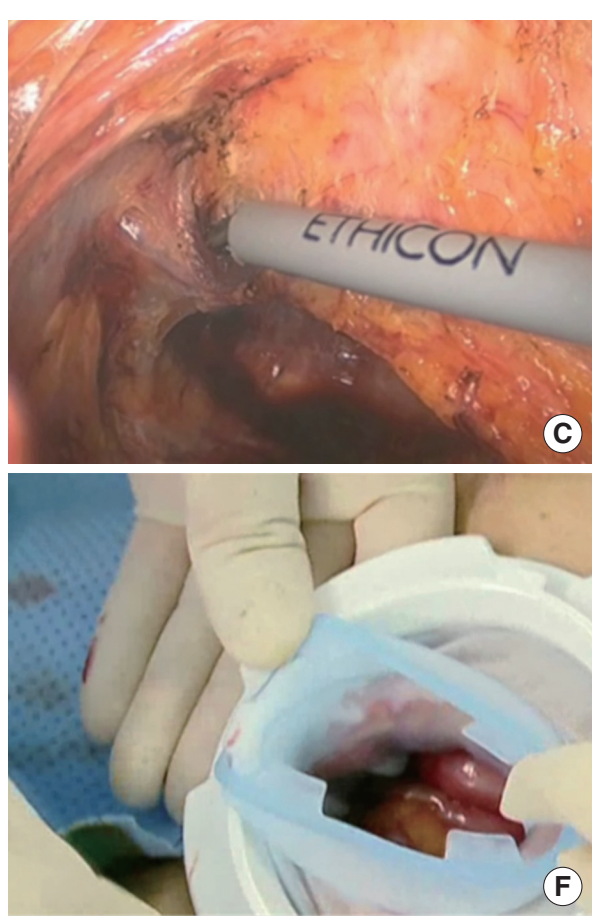

Fig. 2. Reduced-port laparoscopic surgery for treating rectal cancer: (A) medial-to-lateral dissection of the sigmoid and descending colon, and identification of the left ureter and gonadal vessels, (B) high ligation of the inferior mesenteric artery, (C) lateral dissection, (D, E) laparoscopic stapling through the additional port, and (F) extraction of the specimen through the single port as a wound protector. 


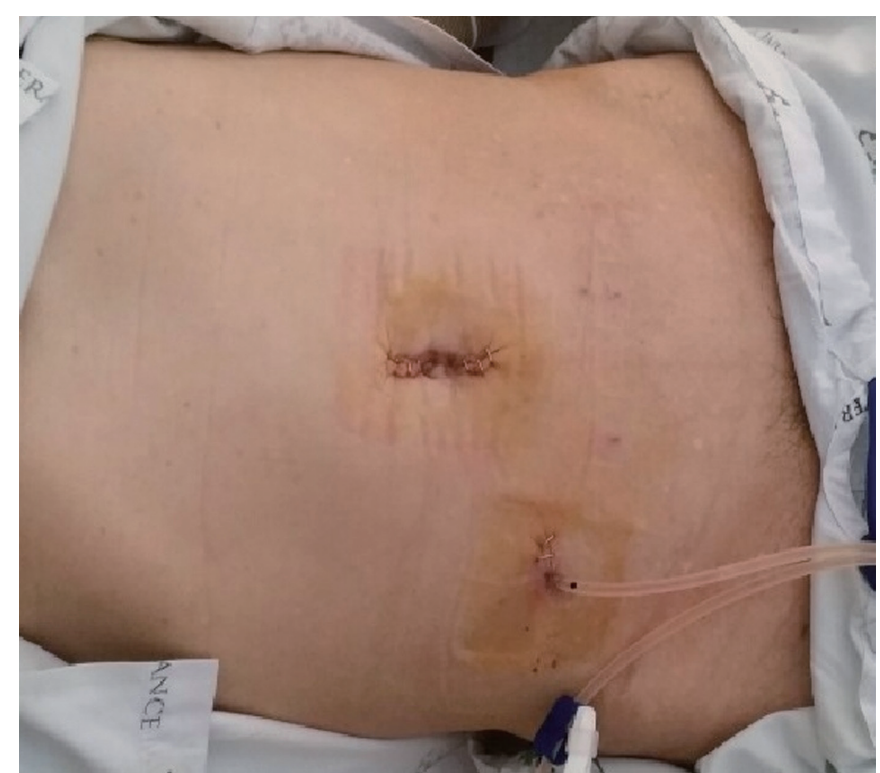

Fig. 3. View of the patient on postoperative day 1 . The additional port site was used for pelvic-drain placement.

time of surgery to ensure an intact anastomosis. At the end of the operation, the additional port site was used for pelvic drain placement (Fig. 3).

\section{RESULTS}

The baseline demographics of patients are tabulated in Table 1. The median age of the 20 patients was 62 years (range, 39-84 years); the patients included 9 women and $11 \mathrm{men}$, and the body mass index was $25.1 \mathrm{~kg} / \mathrm{m}^{2}$ (range, $19.7-29.8 \mathrm{~kg} / \mathrm{m}^{2}$ ). Physical status (ASA classification) was class 1 in six cases, class 2 in twelve, and class 3 in two patients. There were 3 cases (15\%) of rectosigmoid colon cancer, 12 cases (60\%) of upper rectal cancer, and 5 cases $(25 \%)$ of middle rectal cancer. Two of the five patients with middle rectal cancer received neoadjuvant chemoradiotherapy. A history of abdominal surgery was associated with 6 cases (30\%).

All 20 procedures were technically successful without the need for conversion to open surgery (Table 2). However, one patient (5\%) required the insertions of two additional ports because of severe adhesion, and that patient was the only case of conversion from RPLS to multiport laparoscopic surgery (MPLS) based on the definition of conversion given in this study. No patients underwent a planned protective defunctioning ileostomy for distal rectal cancer or had two or more of the risk factors for anastomotic leakage. However, one case of anastomotic leakage was treated by using a transanal repair and a laparoscopic-assisted protective ileostomy. We transected the rectum with one laparoscopic stapler in 17 cases (85\%). The median operation time was 231 minutes (range, 160-347 minutes), and the estimated blood loss was 100 $\mathrm{mL}$ (range, 50-500 mL). The patients convalesced quickly, as eval-
Table 1. Patient characteristics $(\mathrm{n}=20)$

\begin{tabular}{lc}
\hline Characteristic & Value \\
\hline Sex & $11(55)$ \\
Male & $9(45)$ \\
Female & $62(39-84)$ \\
Age (yr) & \\
ASA physical status class & $6(30)$ \\
1 & $12(60)$ \\
2 & $2(10)$ \\
3 & $25.1(19.7-29.8)$ \\
Body mass index (kg/m²) & $3(15)$ \\
Tumor location (distance from anal verge) & $12(60)$ \\
Rectosigmoid colon (>15 cm) & $5(25)$ \\
Upper rectum (11-15 cm) & $2(10)$ \\
Middle (6-10 cm) & \\
Preoperative chemoradiotherapy & $19(95)$ \\
Type of port & $1(5)$ \\
Single port +1 additional port & $6(30)$ \\
Single port +2 additional port & $3.0(0.4-35)$ \\
Previous abdominal surgeries & \\
Preoperative CEA (ng/mL) & \\
\hline
\end{tabular}

Values are presented as number (\%) or median (range).

ASA, American Society of Anesthesiologists; CEA, carcinoembryonic antigen.

uated by using the median time to first flatus passage of 2 days (range, 1-4 days), to soft diet of 4 days (range, 3-6 days), to removal of the urinary catheter of 2 days (range, 1-5 days), and discharge from the hospital of 7 days (range, 5-45 days). The median VAS score on postoperative day 1 was 3 (range, $2-7$ ). No mortalities were associated with the procedure. Seven patients (35\%) developed postoperative complications. One patient developed benign anastomotic stenosis 5 weeks after surgery and was treated by using endoscopic dilation. Paralytic ileus occurred in two patients, and both were responsive to conservative management with intravenous fluids, bowel rest, and nasogastric aspiration. Acute voiding difficulties requiring a urinary catheter occurred in two patients, but those patients subsequently recovered with conservative management. No hernias were observed at the umbilicus and the additional port sites during the follow-up period.

Pathologic characteristics are displayed in Table 3. Five patients (25\%) had stage I tumors, 8 (40\%) had stage II tumors, and 5 (25\%) had stage III tumors. Two of the five patients with middle rectal cancer received neoadjuvant chemoradiotherapy, and one had a ypT0N0M1 (combined pneumonectomy due to lung metastasis) tumor while the other had a ypT3N10 tumor. The median tumor size was $3 \mathrm{~cm}$ (range, 1-7 cm), and the total number of lymph nodes harvested was 16 (range, 7-36). The median proximal and distal resection margins were $10 \mathrm{~cm}$ (range, 7-23 cm) and 


\section{Coloproctology sung Uk Bae, et al.}

Table 2. Perioperative outcomes $(n=20)$

\begin{tabular}{ll}
\hline Outcome & \multicolumn{1}{c}{ Value } \\
\hline Operation time (min) & $231(160-347)$ \\
Blood loss (mL) & $100(50-500)$ \\
No. of stapler firings & \\
1 & $17(85)$ \\
2 & $3(15)$ \\
VAS (score) on POD \#1 & $3(2-7)$ \\
1st Flatus POD (day) & $2(1-4)$ \\
Time to soft diet (day) & $4(3-6)$ \\
Time to removal of the urinary catheter (day) & $2(1-5)$ \\
Hospital days (day) & $7(4-45)$ \\
Conversion & $0(0)$ \\
Morbidity within 30 days after surgery & $7(35)$ \\
Anastomotic leakage & $1(5)$ \\
Anastomosis site stricture & $1(5)$ \\
Ileus & $2(10)$ \\
Voiding difficulty & $2(10)$ \\
Chyle leakage & $1(5)$ \\
Mortality & $0(0)$ \\
\hline
\end{tabular}

Values are presented as median (range) or number (\%).

VAS, visual analogue scales; POD, postoperative day

$2 \mathrm{~cm}$ (range, 1-12 cm), respectively. CRM involvement was found in 1 case (5\%). The median follow-up period was 20 months (range, 12-40 months). No local recurrences were noted, and liver metastasis occurred in 1 patient 10 months after surgery.

\section{DISCUSSION}

Laparoscopic total mesorectal excision for rectal cancer, although it is regarded as technically demanding, offers a better magnified view of the pelvic structures than open surgery and a favorable postoperative short-term recovery, and good long-term oncologic outcomes also have been reported [1-5]. With the development of minimally-invasive surgery, questions of how to minimize surgical trauma and improve cosmetic outcomes have evolved as current topics of active discussion.

Since SILS was first described in 1998 for a laparoscopic appendectomy [6] and cholecystectomy [7], the SILS technique has been incorporated into a variety of laparoscopic procedures, including colectomies [8], adrenalectomies [9], nephrectomies [10], and splenectomies [11]. The potential advantages of this approach are associated with less postoperative incisional pain, less risks for hemorrhage, incisional hernias, and organ injuries, fewer wound complications and improved cosmetic outcomes. Remzi et al. [12] reported the first single-incision laparoscopic right hemicolectomy in 2008. However, the application of the SILS for treating rectal
Table 3. Pathologic characteristics $(\mathrm{n}=20)$

\begin{tabular}{lc}
\hline Characteristic & Value \\
\hline TNM stage & $5(25)$ \\
I & $8(40)$ \\
III & $6(30)$ \\
IV & $1(5)$ \\
Histology & \\
Well differentiated & $5(25)$ \\
Moderately differentiated & $14(70)$ \\
Mucinous & $1(5)$ \\
Tumor size (cm) & $3(1-7)$ \\
Proximal resection margin (cm) & $10(7-23)$ \\
Distal resection margin (cm) & $2(1-12)$ \\
Lymphovascular invasion & $3(15)$ \\
CRM < 1 mm & $1(5)$ \\
Retrieved LNs & $16(7-36)$ \\
\hline
\end{tabular}

Values are presented as number (\%) or median (range).

CRM, circumferential resection margin; LN, lymph node.

cancer has only recently been reported in the published literature [13-16], and the feasibility and the safety of SILS in rectal surgery has not yet been determined because the laparoscopic stapler does not allow a low rectal transection with sufficient distal margins and because rectal traction with laparoscopic instruments from a single port have technical limitations in the narrow bony pelvic cavity.

The present study demonstrates that the reduced laparoscopic TSME offered by using a single port with a multichannel system plus additional port can be performed with good technical efficiency and favorable short-term outcome. In this study, the perioperative outcomes, including the number of days to first gas passing of 2 days (range, 1-4 days), the time to soft diet of 4 days (range, 3-6 days) and a possible hospital stay of 7 days (range, 4-45 days) are comparable to the perioperative outcomes of conventional laparoscopic surgery for rectal cancer $[1,4]$. Although one of the controversies of SILS for the treatment of colorectal cancer is increased operation time when compared with conventional techniques [17], a systematic review of 14 reports showed no significant differences in the operation times between SILS and MPLS for treating colorectal disease [18]. However, SILS for the treatment of rectal cancer is more technically demanding than it is for the treatment of right or sigmoid colon cancer, and data comparing SILS with MPLS for treating rectal cancer are extremely rare. Some previous studies reported average SILS operative times of 229 and 347 minutes $[13,14]$ and an average RPLS operative time of 276 minutes [19]. In the present study, the operative time ranged from 160 to 347 minutes, with a median of 231 minutes for all patients undergoing RPLS. These results show that RPLS may prove to be comparable to MPLS. 
Anastomotic leaks are a dreaded complication in colorectal surgery and a major cause of postoperative morbidity and mortality. Despite adhering to the recommended surgical principles of good blood supply, tension-free anastomosis, low tie (to preserve the left colic artery), and use of a rectal drain (to decrease intraluminal pressure), some anastomoses do leak, especially those placed in low anterior resections [20,21]. Kim et al. [22] suggested that a reduction in the number of linear stapler firings was necessary to avoid anastomotic leakage after a laparoscopic colorectal anastomosis with a double-stapling technique. However, Hamzaoglu et al. [13] reported that the average number of cartridges used was four for TME and six for TSME and commented that an additional 5-mm laparoscope was strongly advised when transecting the colon and the rectum by using endoscopic linear staplers because using the staplers to transect the rectum was the most difficult and time-consuming part of the operation. For appropriate endo-stapling, especially in patients with rectal cancer, the angle between the bowel and the cartridge of the stapler needs to be 90 degrees. While it is difficult to make a right angle between the bowel and the cartridge of the stapler in pure SILS, RPLS for treating rectal cancer can facilitate appropriate endo-stapling with triangulation in a narrow pelvic cavity. In this study, RPLS made it possible to transect the rectum with only one laparoscopic stapler in 17 patients, with only one case (5\%) of anastomotic leakage. The authors believe that the adoption of the RPLS for treating rectal cancer can facilitate appropriate endo-stapling after a TSME.

Although the role of pelvic drainage in the prevention of anastomotic leakage after rectal cancer surgery is controversial, we routinely put a drain in the pelvic cavity. Changes in the color and the odor of the drain content have generally been assumed to suggest an anastomotic leak, and drainage after a rectal anastomosis can help to evacuate residual effusions. Tsujinaka et al. [23] reported that changes in the drain content that suggested an anastomotic leak were observed in 15 patients $(71.4 \%)$ and that anastomotic leaks were resolved by using conservative treatment with the existing drain in 10 patients (47.6\%). While drainage at the right iliac fossa cannot be applied to SILS, the incision of the additional port can be used for pelvic drain placement in RPLS.

The present study shows that RPLS for TSME did not compromise the oncologic efficacy for the treatment of low rectal cancer. One of the most important parameters that express the quality of a total mesorectal excision are the rate of CRM involvement and the number of lymph nodes harvested. In the CLASICC (Conventional vs. Laparoscopic- Assisted Surgery in Colorectal Cancer) trial [24], the CRM involvement rate was $12 \%$ in the laparoscopic group and $6 \%$ in the open group, and the COLOR (COlorectal cancer Laparoscopic or Open Resection) II trial [1] resulted in negative margins in 545 of 588 patients (93\%) in the laparoscopic group and 274 of 300 patients (91\%) in the open group. In the current study, CRM involvement was found in 1 case (5\%), and the median number of lymph nodes harvested was 16: these data are acceptable compared with previous studies. Additionally, during the median follow-up period of 20 months, there were no local recurrences. We believe that appropriate triangulation with tissue retraction and dissection played an important role in the oncologic safety.

In the current study, one patient (5\%) required additional insertions of two ports because dissection in the narrow and deep pelvic space is extremely difficult. Hirano et al. [19] also reported that one patient $(6.3 \%)$ required the insertions of an additional two ports during RPLS. It is important to keep in mind that the use of additional ports or instruments is not a failure of SILS or RPLS. Although we do not have any experience with needlescopic surgery, mini-laparoscopic instruments with an outer diameter of $2.3 \mathrm{~mm}$, which has an integrated delivery conduit allowing direct percutaneous placement into the abdomen, may be used as a modified reduced-port laparoscopic approach to performing a TME [25]. This technique should lead to cosmetic advantages that are similar to those of SILS or RPLS and to better ergonomics with laparoscopic instruments. Conversion to hand-assisted surgery, which is still a minimally-invasive platform that enlarges the incision length from 7 to $7.5 \mathrm{~cm}$, may lead to an avoidance of open conversion.

SILS has its own unique challenges, including the relative loss of triangulation due to straight instruments being parallel to the laparoscope, the in-line vision, the clashing of instruments, and the steep learning curve for mastering the procedure. However, in the current study, the problems anticipated for the loss of triangulation in the limited space in using an additional port were overcome. Additionally, a laparoscope with a flexible tip may be helpful for gaining triangulation and a better operation to overcome the technical challenges of SILS or RPLS. Hamzaoglu et al. [13] proposed to use an articulated laparoscopic instrument and to elevate the sigmoid colon by using percutaneously inserted stitches, which could be helpful for retraction, and Leroy et al. [26] reported a laparoscopic sigmoidectomy using extracorporeal magnets for adequate exposure. Uematsu et al. [27] introduced a suspending bar and an extracorporeal magnet to lift and retract the rectum.

Our study has several limitations, including its retrospective nature, the small size of the study, the absence of low rectal cancer, and the lack of long-term data on cosmesis for single-port versus multiport surgery. From limited information at a single center, we can suggest the following advantages of RPLS for treating colorectal cancer: (1) maintenance of the cosmetic advantages of SILS, (2) improved visualization and decreased collisions between the laparoscopic instruments and the camera, (3) ability to perform a rectal transaction by using a laparoscopic endostapler with sufficient distal margins, and (4) ability to use an additional incision for an additional port for pelvic-drain placement.

In conclusion, RPLS for a TSME in treating colorectal cancer is technically feasible and safe and does not compromise oncologic safety. However, further studies comparing RPLS to a conventional laparoscopic low anterior resection are needed to prove the advantages of the RPLS procedure. 


\section{CONFLICT OF INTEREST}

No potential conflict of interest relevant to this article was reported.

\section{REFERENCES}

1. Color II Study Group, Buunen M, Bonjer HJ, Hop WC, Haglind E, Kurlberg G, et al. COLOR II. A randomized clinical trial comparing laparoscopic and open surgery for rectal cancer. Dan Med Bull 2009;56:89-91.

2. Kang SB, Park JW, Jeong SY, Nam BH, Choi HS, Kim DW, et al. Open versus laparoscopic surgery for mid or low rectal cancer after neoadjuvant chemoradiotherapy (COREAN trial): short-term outcomes of an open-label randomised controlled trial. Lancet Oncol 2010;11:637-45.

3. Lujan J, Valero G, Hernandez Q, Sanchez A, Frutos MD, Parrilla P. Randomized clinical trial comparing laparoscopic and open surgery in patients with rectal cancer. Br J Surg 2009;96:982-9.

4. Green BL, Marshall HC, Collinson F, Quirke P, Guillou P, Jayne DG, et al. Long-term follow-up of the Medical Research Council CLASICC trial of conventional versus laparoscopically assisted resection in colorectal cancer. Br J Surg 2013;100:75-82.

5. Bagshaw PF, Allardyce RA, Frampton CM, Frizelle FA, Hewett PJ, McMurrick PJ, et al. Long-term outcomes of the australasian randomized clinical trial comparing laparoscopic and conventional open surgical treatments for colon cancer: the Australasian Laparoscopic Colon Cancer Study trial. Ann Surg 2012;256:915-9.

6. Esposito C. One-trocar appendectomy in pediatric surgery. Surg Endosc 1998;12:177-8.

7. Piskun G, Rajpal S. Transumbilical laparoscopic cholecystectomy utilizes no incisions outside the umbilicus. J Laparoendosc Adv Surg Tech A 1999;9:361-4.

8. Ramos-Valadez DI, Patel CB, Ragupathi M, Bartley Pickron T, Haas EM. Single-incision laparoscopic right hemicolectomy: safety and feasibility in a series of consecutive cases. Surg Endosc 2010; 24:2613-6.

9. Chung SD, Huang CY, Wang SM, Tai HC, Tsai YC, Chueh SC. Laparoendoscopic single-site (LESS) retroperitoneal adrenalectomy using a homemade single-access platform and standard laparoscopic instruments. Surg Endosc 2011;25:1251-6.

10. Raman JD, Bagrodia A, Cadeddu JA. Single-incision, umbilical laparoscopic versus conventional laparoscopic nephrectomy: a comparison of perioperative outcomes and short-term measures of convalescence. Eur Urol 2009;55:1198-204.

11. Targarona EM, Pallares JL, Balague C, Luppi CR, Marinello F, Hernandez P, et al. Single incision approach for splenic diseases: a preliminary report on a series of 8 cases. Surg Endosc 2010;24: 2236-40.

12. Remzi FH, Kirat HT, Kaouk JH, Geisler DP. Single-port laparos- copy in colorectal surgery. Colorectal Dis 2008;10:823-6.

13. Hamzaoglu I, Karahasanoglu T, Baca B, Karatas A, Aytac E, Kahya AS. Single-port laparoscopic sphincter-saving mesorectal excision for rectal cancer: report of the first 4 human cases. Arch Surg 2011; 146:75-81.

14. Bulut O, Nielsen CB, Jespersen N. Single-port access laparoscopic surgery for rectal cancer: initial experience with 10 cases. Dis Colon Rectum 2011;54:803-9.

15. Hua-Feng P, Zhi-Wei J, Gang W, Xin-Xin L, Feng-Tao L. A novel approach for the resection of low rectal cancer. Surg Laparosc Endosc Percutan Tech 2012;22:537-41.

16. van den Boezem PB, Sietses C. Single-incision laparoscopic colorectal surgery, experience with 50 consecutive cases. J Gastrointest Surg 2011;15:1989-94.

17. Champagne BJ, Lee EC, Leblanc F, Stein SL, Delaney CP. Singleincision vs straight laparoscopic segmental colectomy: a case-controlled study. Dis Colon Rectum 2011;54:183-6.

18. Zhou YM, Wu LP, Zhao YF, Xu DH, Li B. Single-incision versus conventional laparoscopy for colorectal disease: a meta-analysis. Dig Dis Sci 2012;57:2103-12.

19. Hirano Y, Hattori M, Douden K, Shimizu S, Sato Y, Maeda K, et al. Single-incision plus one port laparoscopic anterior resection for rectal cancer as a reduced port surgery. Scand J Surg 2012;101: 283-6.

20. Xiao L, Zhang WB, Jiang PC, Bu XF, Yan Q, Li H, et al. Can transanal tube placement after anterior resection for rectal carcinoma reduce anastomotic leakage rate? A single-institution prospective randomized study. World J Surg 2011;35:1367-77.

21. Dworkin MJ, Allen-Mersh TG. Effect of inferior mesenteric artery ligation on blood flow in the marginal artery-dependent sigmoid colon. J Am Coll Surg 1996;183:357-60.

22. Kim JS, Cho SY, Min BS, Kim NK. Risk factors for anastomotic leakage after laparoscopic intracorporeal colorectal anastomosis with a double stapling technique. J Am Coll Surg 2009;209:694701.

23. Tsujinaka S, Kawamura YJ, Konishi F, Maeda T, Mizokami K. Pelvic drainage for anterior resection revisited: use of drains in anastomotic leaks. ANZ J Surg 2008;78:461-5.

24. Jayne DG, Guillou PJ, Thorpe H, Quirke P, Copeland J, Smith $\mathrm{AM}$, et al. Randomized trial of laparoscopic-assisted resection of colorectal carcinoma: 3-year results of the UK MRC CLASICC Trial Group. J Clin Oncol 2007;25:3061-8.

25. Ng KS, Kuo S. Are we entering a needlescopic era? ANZ J Surg 2012;82:864-5.

26. Leroy J, Cahill RA, Peretta S, Marescaux J. Single port sigmoidectomy in an experimental model with survival. Surg Innov 2008; 15:260-5.

27. Uematsu D, Akiyama G, Narita M, Magishi A. Single-access laparoscopic low anterior resection with vertical suspension of the rectum. Dis Colon Rectum 2011;54:632-7. 